Original Research Paper

\title{
Prevalence and Risk Factors Associated with Needle Sticks and Sharp Injuries among Nurses at the Accident and Emergency Department of Komfo Anokye Teaching Hospital, Kumasi-Ghana
}

\author{
${ }^{1}$ Augustine Kumah, ${ }^{2}$ Hilda Agortimevor, \\ ${ }^{3}$ Francis Akakpo, ${ }^{4}$ Hope Akpeke, ${ }^{5}$ Gideon Azi and ${ }^{6}$ Etornam Gblende \\ ${ }^{1}$ Department of Community Health, \\ Centre of Excellence in Reproductive Health Innovation (CERHI), University of Benin, Nigeria \\ ${ }^{2}$ Department of Accident and Emergency, Sacred Heart Hospital, Abor-Weme, Ghana \\ ${ }^{3}$ Department of Health Informatics, Nursing Training College, Ho, Ghana \\ ${ }^{4}$ Department of Nursing, University of Ghana, Legon, Ghana \\ ${ }^{5}$ Department of Mental Health, Nursing Training College, Ho, Ghana \\ ${ }^{6}$ Department of Mental Health, Mental Health Authority, Accra, Ghana
}

Article history

Received: 26-10-2020

Revised: 18-12-2020

Accepted: 28-12-2020

Corresponding Author:

Augustine Kumah

Department of Community

Health, Centre of Excellence in

Reproductive Health Innovation

(CERHI), University of Benin,

Nigeria

Email: augustinekumah@gmail.com

\begin{abstract}
Needle Sticks and Sharps Injuries (NSSI) represent a major occupational hazard in the health care industry, with professional nurses incurring a large proportion of the total burden particularly with items that have been previously used on patients. This study investigated the prevalence and risk factors associated with needle sticks and sharp injuries among nurses at the accident and emergency department of Komfo Anokye Teaching Hospital, Kumasi-Ghana. A cross-sectional quantitative descriptive survey was conducted among 113 Nurses at Accident \& Emergency department of Komfo Anokye Teaching Hospital in Ghana using a simple random sampling in selecting participants. A structured questionnaire was used to collect data from the participants. The study shown a prevalence of $47 \%$ needle stick and sharp injury among nurses with $33.6 \%$ of those injuries resulting from needle stick. Majority $(25.5 \%)$ of the respondents allowed the injury to bleed as an initial intervention measure. Majority $(68.1 \%)$ reported the incidence of the injury while $(31.9 \%)$ did not. Majority $(33.3 \%)$ of them did not report because they did not know who to report to. Work pressure $(53.1 \%)$ was identified as the major contributing factor for needle stick and sharp injuries. The study shown a prevalence of $47 \%$ needle stick and sharp injury among nurses with $33.6 \%$ of those injuries resulting from needle stick. Nurses should be trained on safety measures of handling and disposing needles and sharps and should also be encouraged to report NSSIs in other to get the right treatment and counselling.
\end{abstract}

Keywords: Ghana, Needle Sticks, Nurses, Prevalence, Risk Factors

\section{Introduction}

Needle Sticks and Sharps Injuries (NSSIs) are common occupational injuries among health care workers (van der Molen et al., 2011; Kebede et al., 2012). Needle sticks and sharps injuries are wound caused by needles and other sharp medical devices that accidentally pierce or cut the skin. Such injuries though small can be dangerous because these sharps and needles have come into contact with blood and other body fluids and they may carry the risk of infections. HIV and hepatitis infection can be contracted through NSSIs. Injuries with NSSIs involve needles, scalpels, lancets, razor blade, scissors, metal wire, retractors, clamps, pins, staples, cutters and glass items. Health Care Workers (HCWs), including the nurses, doctors, dentist, operating departments, laboratory workers, phlebotomist, cleaners and laundry workers, are at risk of injuries with 
sharps (European Agency for Safety and Health at Work, 2009). Among all the health professional groups, the nursing staff had been identified as the highest at risk for sharps-related injuries (Kable et al., 2011; Kumar et al., 2012; Adib-Hajbaghery and Lotfi, 2013). Nurses had a large percentage of the total burden of NSSIs especially with items that had been earlier used on patients (Clarke et al., 2002; Ayranci and Kosgeroglu, 2004; Smith et al., 2006).

World Health Organization (WHO) reports had addressed the risk factors in accordance with earlier reviews, on NSSIs, to include, the lack of engineering controls to ensure safer needle devices, insufficient hospital staffing and recapping of needles after use (Wilburn and Eijkemans, 2004; Prüss-Üstün et al., 2005). The International Healthcare Worker Safety Center, in the US, had shown that, injections and drawing of venous blood accounted for 23.6 and $11.5 \%$ of NSSIs, respectively (Perry et al., 2003). Registered Nurses (RN) working on patient care units with lower staffing rates and higher levels of emotional fatigue related to their jobs also had significantly greater possibilities of NSSIs (Clarke et al., 2002). Nursing practices that increased the risk of needle stick injury were often connected with activities such as recapping of needles, failing to dispose of used needles properly, collision with other workers and hidden needles in bed linens (Kumar et al., 2012). In sub-Saharan Africa, only few studies had examined the occurrence and risk of sharps injuries among nursing staffs (Nsubuga and Jaakkola, 2005); Amira and Awobusuyi, 2014). Comparing nurses with the physicians, nurses had highest risk of needle stick and sharps injury (Memish et al., 2013). Ancillary staffs were also at risk of NSSIs, because of their involvement in handling items contaminated with blood, (Amira and Awobusuyi, 2014).

In Ghana, data collection on NSSIs was inadequate (Kommogldomo, 2016). Under reporting was a major challenge. As a result, authorities were unable to quantify exposure level for policymaking. There was also lack of data to be used for the purpose of prevention and in the case of later infection (Rais and Jamil, 2013). Low reporting of NSIs had been attributed to lack of awareness and perceived low risk of transmission of infection, (Chalya et al., 2015). This study therefore assessed the risk factors associated with needle sticks and sharp injuries among nurses at the accident and emergency department of Komfo Anokye Teaching Hospital in Ghana.

\section{Methods}

A cross-sectional quantitative descriptive survey was conducted among 113 Nurses at Accident and Emergency department of Komfo Anokye Teaching
Hospital in Ghana between August and October 2018 using a convenient sampling technique in selecting participants. The sample size was estimated using Leslie Kish formula with a prevalence of $50 \%(\mathrm{P}=0.5)$ and a margin of error of $5 \%$ at a $95 \%$ confidence interval. All Nurses, who were on duty, were included in the study. Nurses who were off duty, on leave and not present at the time of the study were excluded from the study. A pilot tested predesigned validated questionnaire was used to collect data from the respondents. Results were analyzed using SPSS version 20 and data presented using tables and graphs.

\section{Results}

\section{Socio-Demographic Characteristics of Respondents}

The Table 1 shows findings on the socio-demographic characteristics of respondents.

Majority $(56.6 \%)$ of the respondents were female and the mean age was 26. 23. Majority (53.1\%) were married and $(46.9 \%)$ were single. Registered general nurses form a simple majority of the study (54.0\%), Emergency nurses were $(26.5 \%)$ and $(19.5 \%)$ were enrolled nurses (Fig. 1).

The study shown that majority $60(53 . \%)$ of the respondents never had a needle stick and sharp injuries while $53(47 . \%)$ of respondents ever had a needle stick and sharp injuries (Fig. 2).

Table 1: Socio-demographic characteristics of respondents (n =113)

\begin{tabular}{lcc}
\hline Responses & Frequency & Percent \\
\hline Gender & 49 & \\
Male & 64 & 43.4 \\
Female & 5 & 56.6 \\
Age & 41 & \\
20-24 years & 44 & 4.4 \\
26-30 years & 20 & 36.3 \\
31-35 years & 3 & 38.9 \\
36-40 years & & 17.7 \\
Above 40 years & 53 & 2.7 \\
Marital Status & 60 & \\
Single & & 46.9 \\
Married & 61 & 53.1 \\
Nurses Group & 30 & \\
Registered general nurse & 22 & 54.0 \\
Emergency nurse & & 26.5 \\
Enrolled nurse & 37 & 19.5 \\
Length of Service & 62 & 32.7 \\
1-5 years & 9 & 54.9 \\
6-10 years & 5 & 8.0 \\
11-15 years & & 4.4 \\
More than 15 years & 77 & 68.2 \\
Hours at work per shift & 26 & 23.0 \\
8 h & 10 & 8.8 \\
12 h & & \\
More than 12 h & & \\
\hline & & \\
& &
\end{tabular}




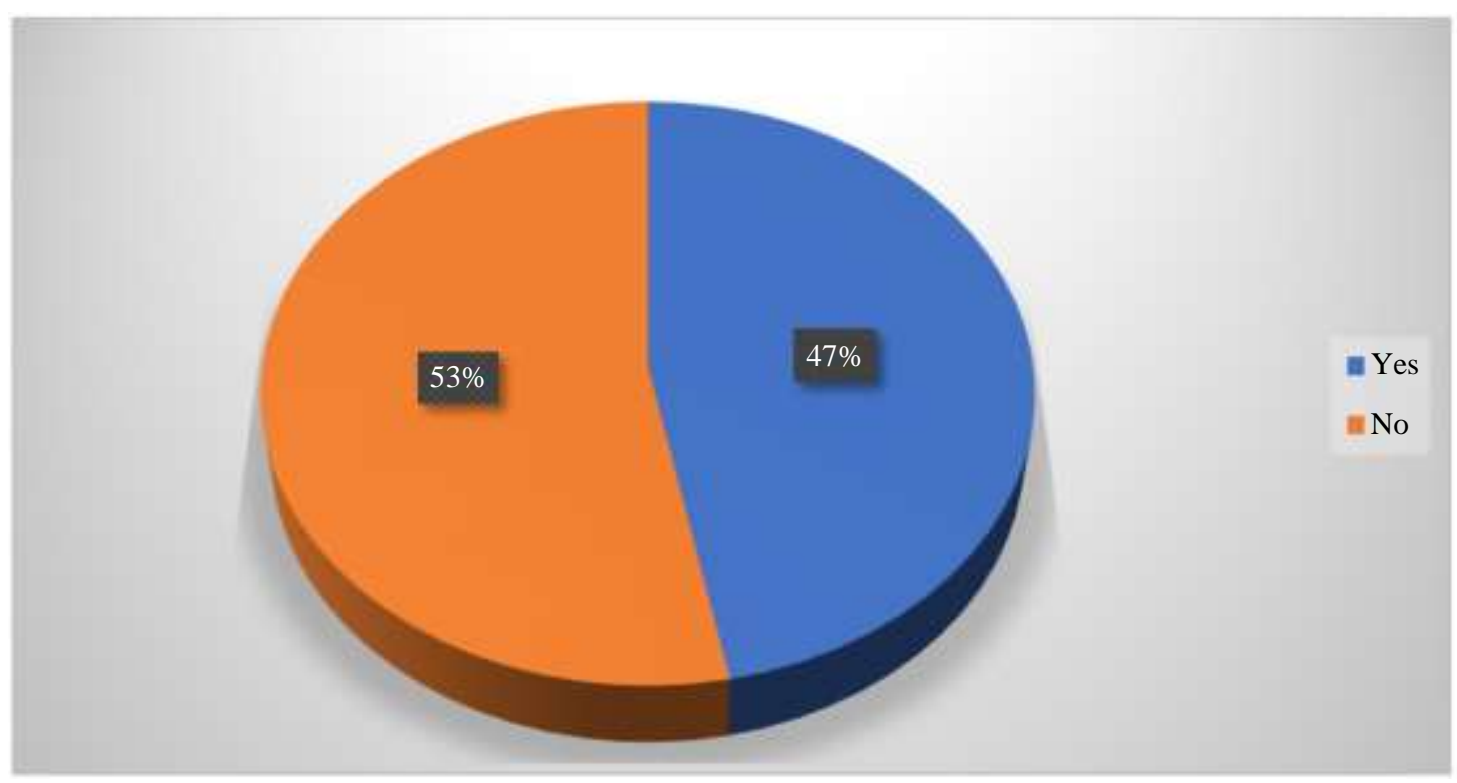

Fig. 1: Prevalence of NSSIs among nurses at Komfo Anokye Teaching Hospital

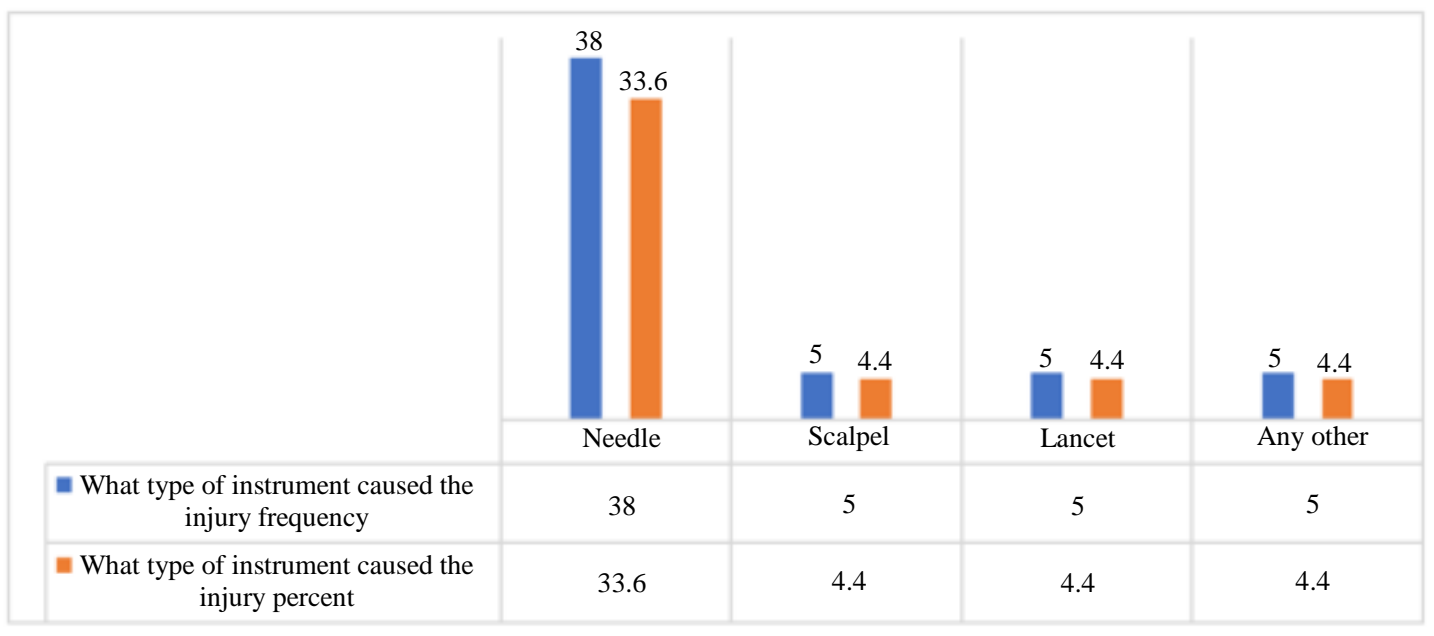

Fig. 2: Source of needle stick and sharp injury among nurses at Komfo Anokye Teaching Hospital

Out of the total number (53) of respondents who ever had a needle stick and sharp injuries, 38 (33.6\%) of those injuries were from needle sticks.

Measures taken following needle stick and sharp injury among Nurse at Komfo Anokye Teaching Hospital.

The study shown that most of the respondents 12 (25.5\%) allowed the injury to bleed after a needle stick or sharp injury as the initial intervention measure while $11(23.4 \%)$ washed the injury site with soap and water, 9 $(19.1 \%)$ notified the infection control office of the hospital and $5(10.6 \%)$ applied antiseptic agent to the site. However, $10(21.3 \%)$ of the respondents allowed the injury to bleed for some time, then washed the injury site with soap and water, later applied antiseptic agent to the site before notifying the infection control office of the hospital (Fig. 3).

Out of the total percentage of respondents of 60 who have ever had a needle stick and sharp injuries, majority $32(68.1 \%)$ reported the incidence of the injury while 15 (31.9\%) of respondents did not (Table 2 ).

The study looked at reasons why some of the nurse do not report needle stick and sharp injuries. Out of the total percentage $(39.1 \%)$ of respondent who have ever had needle stick and sharp injuries, $(33.3 \%)$ of them did not report the incidence of the injury because they did not know who to report to. Also, a proportion (26.7\%) of the respondent did not report because of the fear of stigma and $13.3 \%$ did not report because they felt it was not important. 


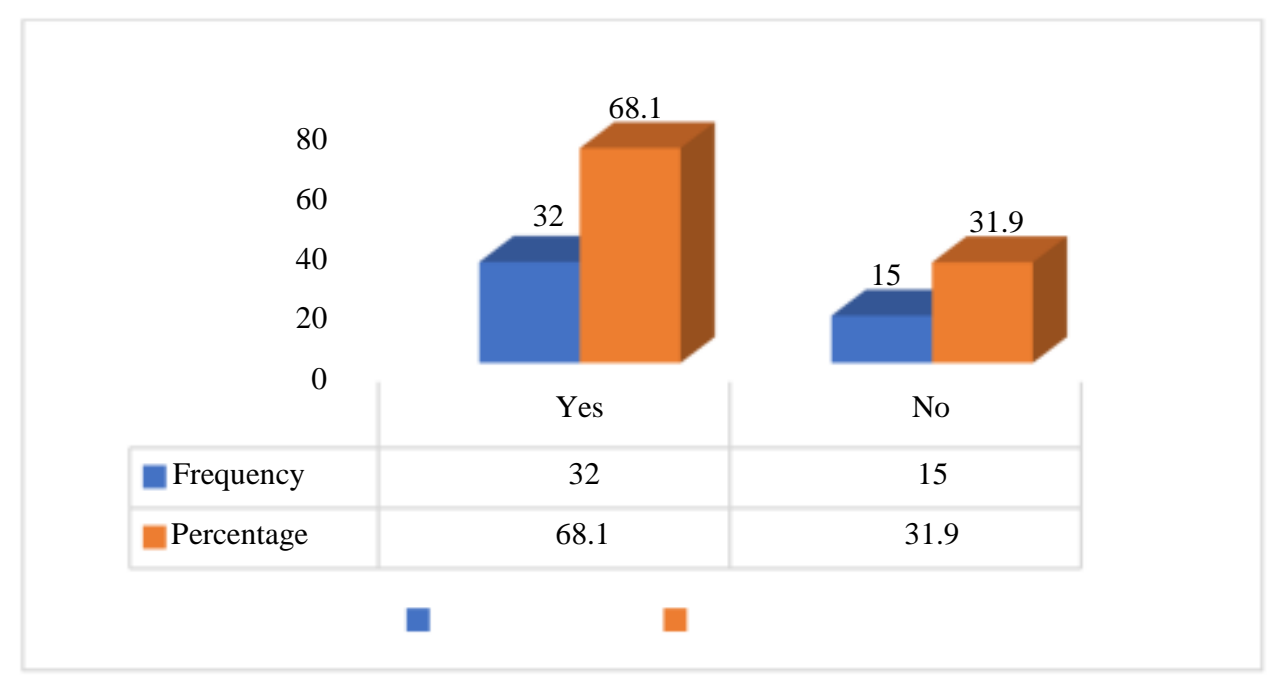

Fig. 3: Reporting rate of needle stick and sharp injuries among Nurses at Komfo Anokye Teaching Hospital

Table 2: Reasons for not reporting needle stick and sharp injuries among Nurses at Komfo Anokye Teaching Hospital

\begin{tabular}{lcc}
\hline Response & Frequency & Percent \\
\hline Did not know whom to report to & 5 & 33.3 \\
I do not think it was important to report & 2 & 13.3 \\
Fear of stigma & 4 & 26.7 \\
Thought patient was at low risk for HIV infection & 1 & 6.7 \\
Belief my vaccination status is sufficient & 2 & 13.3 \\
Lack of knowledge of appropriate procedure after injury & 1 & 6.7 \\
Total & 15 & 100.0 \\
\hline
\end{tabular}

Table 3: Contributing factor(s) of needle stick and sharps injuries among Nurses at Komfo Anokye Teaching Hospital

\begin{tabular}{lrr}
\hline Response & Frequency (n) & Percent (\%) \\
\hline Fatigue & 10 & 8.8 \\
Work Pressure & 60 & 53.1 \\
Non co-operative/restless clients & 10 & 8.8 \\
Unsafe medical sharps & 5 & 4.4 \\
Unclear work procedures & 16 & 3 \\
Work overload & 7 & 2.7 \\
Indiscriminate needles and sharps disposal by some nurses & 2 & 6.2 \\
Lack of access and failure to use sharps containers immediately after injection & 113 & 1.8 \\
Total (n) & & 100.0 \\
\end{tabular}

Table 3 above shows contributing factor (s) of needle stick and sharps injuries among Nurses at Komfo Anokye Teaching Hospital. The study identified work pressure $(53.1 \%)$ as the major contributing factor for needle stick and sharp injuries.

\section{Discussion}

Needle stick and sharp injuries were among the most common occupational injuries in HCWs that comprise about $12 \%$ of all working people worldwide. WHO study reported that the global burden of NSIs average to 0.2-4.7 NSI per HCW annually, which was approximately three million HCWs affected yearly (Prüss-Üstün et al., 2005). Among HCWs, the highest incidence of these injuries had been reported among nurses. This was shown in a study that reported $52.8 \%$ of nurses being victims of needle stick and sharp injuries from daily administration of injections (Fourie and Keogh, 2011). Various international studies had investigated the incidence of NSIs and had shown the occurrence varies between different countries, with NSIs more prevalent in developing countries. Also, the exact number of NSIs was undefined due to the lack of reporting (Memish et al., 2013); NSIs recorded through standard occupational recording systems can underrate the true incidence by ten times the amount (Memish et al., 2013). WHO reports revealed the number of sharps and needle injures per person among healthcare workers in Africa, Western Mediterranean and Asia to be 4 per year, a low data compared with developed countries such as the United States and Europe (Prüss-Üstün et al., 2005). 
A study conducted in western Tanzania, among HCWs at a tertiary care hospital, found high rates of NSIs among nurses $(71.0 \%)$ and it occurred frequently in the Accident and Emergency department (33.3\%), (Chalya et al., 2015; Lori et al., 2016) conducted a study among nurses at the A\&E of KATH and found a high rate of sharps related injuries among the nurses.

Ghana like many developing nations do not have empirical national statistics on needle stick injuries even though some individuals might have done some works in that area (Kommogldomo, 2016). These statistics are however important for policies on occupational health exposure of healthcare workers. The Occupational Health and Safety Policy Guidelines for the health sector of Ghana came into existence in June 2010; therefore, it is not farfetched that there is not enough data on occupational health exposures.

\section{Prevalence of NSSIs Among Nurses at Komfo Anokye Teaching Hospital}

In this study, the prevalence of NSSIs is $47 \%$ among nurses at the KATH. Having such high prevalence of needle stick amongst nurses means that, these nurses are at risk of occupationally acquired bloodborne diseases (such as hepatitis B and C and HIV) as the result of Needlestick and Sharps Injuries (NSIs). The high prevalence of needle sticks amongst nurses at KATH might be due to the work pressure because of the excessive number of patients per nurse since KATH is a teaching hospital and a referral facility for all hospitals in the Ashanti and its neighboring regions within the middle belt of Ghana. The result of this study is slightly different from the results of (Lee and Hassim, 2005) which shown a prevalence of $50.7 \%$ of needle sticks among nurses in a General Hospital in Malaysia.

\section{Source of Needle Stick and Sharp Injury Among Nurses}

This study also shown that needles are the major source of needle stick and sharp injuries among nurses at KATH. So many reasons may have accounted for this; nurses recapping used needles before disposal, improper handling and disposal of needles by nurses or nurses unable to disposed off used needles promptly after use. This result is in line with the findings of (Lori et al., 2016) which found needles as the major source of needle stick and sharp injuries among Nurses.

On the measures taken by nurses during needle stick injuries, the study shown that nurses with needle stick or sharp injury either allowed the injury to bleed, washed the injury site with soap and water, notified the infection control office of the hospital or applied antiseptic agent to the site after injury occurred. This is similar to studies of (Manzoor et al., 2010; Muralidhar et al., 2010; Jahangiri et al., 2016) which shown that nurses cleaned the needlestick wound after the injury and washed the wound with soap and water.

\section{Reporting Rate of Needle Stick and Sharp Injuries Among Nurses}

The study however shown that $(33.3 \%)$ of nurses did not report the incidence of needle stick or sharp injuries because they did not know who to report to. This might be because safety protocols on needle sticks and sharp injuries and who to call might not be displayed in the wards as recommended by the national guideline on needle stick and sharp injuries. This finding is similar to that of (Muralidhar et al., 2010) which found that healthcare workers did not report the injuries as they did not know where to report. Others also do not because of either the fear of stigma, felt it wasn't important, believed they were protected by their vaccination status, thought patient were at low risk of HIV infection or lack appropriate procedure to follow after the injury. It is important for all nurses and healthcare worker to immediately report all work-related needlestick injuries and cuts from sharp objects particularly those that are contaminated with another person's blood or other potentially infectious material.

\section{Contributing Factor(s) of Needle Stick and Sharps Injuries among Nurses}

Needle stick and sharps injuries are usually caused by simple and preventable mistakes in handling needles and sharp devices. Needle stick injury can occur even before use, during use, after use, before disposal and during or after disposal of the needle or sharp instrument. However, most of these injuries happen after use and before or during disposal process by which time the needle or sharp instrument was already contaminated hence posed the risk of transmission of infectious diseases (Bhardwaj et al., 2014). The study identified Fatigue, Pressure, Non co-operative/restless clients, Unsafe medical sharps, Unclear work procedures, Work overload, indiscriminate needles and sharps disposal by some nurses, Lack of access and failure to use sharps containers immediately after injection as contributing factors to needle stick and sharp injuries. This finding is in line with the findings of (Ilhan et al., 2006; Clarke, 2007; European Agency for Safety and Health at Work, 2009; Gorman et al., 2014) which also found that these factors are interconnected and thus do not occur in isolation.

\section{Conclusion}

This study showed that Nurses at KATH are at high risk of NSSIs. This is shown by the high prevalence of NSSIs among the nurses. Needle sticks are major source of NSSIs among nurses at KATH. The rate of underreporting of NSSIs is also high among nurses who 
had needle stick injuries. Work pressure was identified as the major contributing factor for needle stick and sharp injuries among nurses at KATH.

\section{Recommendation}

The Public Health division of the hospital should provide sufficient safety boxes for disposing sharps and needles. Secondly more nurses should be employed to reduce the workloads on nurses at work as work overload contributes to needle sticks injuries. Protective equipment should be provided in sufficient quantities to minimize the effects of injuries on nurses. There should be regular awareness campaign and orientation for newly employed nurses on needle stick and sharp injuries and its preventive measures. Nurses should be encouraged to report NSSIs in other to get the right treatment and counselling. This will enhance data collection on NSSIs and will be a contributing factor to help in policy making. With the high prevalence of NSSIs among nurse at $\mathrm{KATH}$, it will be appropriate for nurses to undergo health screening at regular intervals.

\section{Acknowledgement}

We would like to thank all the study participants and the Nursing Administration of the Komfo Anokye Teaching Hospital, Kumasi.

\section{Author's Contributions}

Augustine Kumah: Coordinated the entire study, data analysis, discussion and the writing of the manuscript and its review.

Hilda Agortimevor: Coordinated the Literature review contributed to the discussion and writing of the manuscript.

Francis Akakpo: Coordinated the data-analysis and contributed to the discussion and writing of the manuscript.

Hope Akpeke: Coordinated the data collection, data entering and contributed to the data analysis and the writing of the manuscript.

Gideon Azi: Contributed to the data Collection and the writing of the manuscript.

Etornam Gblende: Contributed to the Literature review, data analysis and the writing of the manuscript.

\section{Ethics}

Ethical approval was obtained from the Ghana Health Service Ethical and the Kwame Nkrumah University of Science and Technology (KNUST) Ethical Review committees. However, local permission was obtained from the Hospital management of the Komfo Anokye Teaching Hospital, Kumasi and its research ethics committee. The main ethical issues related to the protection of participant information, consent, autonomy and confidentiality. Consent was obtained from the participants. Participation was voluntary and respondents could withdraw from the study at any stage without any penalty.

\section{References}

Adib-Hajbaghery, M., \& Lotfi, M. S. (2013). Behavior of healthcare workers after injuries from sharp instruments. Trauma monthly, 18(2), 75.

Ayranci, U., \& Kosgeroglu, N. (2004). Needlestick and sharps injuries among nurses in the healthcare sector in a city of western Turkey. Journal of Hospital Infection, 58(3), 216-223.

Amira, C. O., \& Awobusuyi, J. O. (2014). Needle-stick injury among health care workers in hemodialysis units in Nigeria: a multi-center study. Int J Occup Environ Med (The IJOEM), 5(1 January), 228-1.

Bhardwaj, A., Sivapathasundaram, N., Yusof, M. F., Minghat, A. H., Swe, K. M. M., \& Sinha, N. K. (2014). The prevalence of accidental needle stick injury and their reporting among healthcare workers in orthopaedic wards in general hospital Melaka, Malaysia. Malaysian orthopaedic journal, $8(2), 6$.

Chalya, P. L., Seni, J., Mushi, M. F., Mirambo, M. M., Jaka, H., Rambau, P. F., ... \& Kalluvya, S. E. (2015). Needle-stick injuries and splash exposures among health-care workers at a tertiary care hospital in north-western Tanzania. Tanzania Journal of Health Research, 17(2).

Clarke, S. P. (2007). Hospital work environments, nurse characteristics and sharps injuries. American Journal of Infection Control, 35(5), 302-309.

Clarke, S. P., Sloane, D. M., \& Aiken, L. H. (2002). Needlestick injuries to nurses, in context. LDI issue brief, 8(1), 1 .

European Agency for Safety and Health at Work. (2009). New and emerging risks in occupational safety and health. European Agency for Safety and Health at Work.

Fourie, W. J., \& Keogh, J. J. (2011). The need for continuous education in the prevention of needlestick injuries. Contemporary nurse, 39(2), 194-205.

Gorman, T., Dropkin, J., Kamen, J., Nimbalkar, S., Zuckerman, N., Lowe, T., ... \& Freund, A. (2014). Controlling health hazards to hospital workers: A reference guide. NEW SOLUTIONS: A Journal of Environmental and Occupational Health Policy, 23(1_suppl), 1-169.

Ilhan, M. N., Durukan, E., Aras, E., Türkçüoğlu, S., \& Aygün, R. (2006). Long working hours increase the risk of sharp and needlestick injury in nurses: the need for new policy implication. Journal of advanced nursing, 56(5), 563-568. 
Jahangiri, M., Rostamabadi, A., Hoboubi, N., Tadayon, N., \& Soleimani, A. (2016). Needle stick injuries and their related safety measures among nurses in a university hospital, Shiraz, Iran. Safety and Health at work, 7(1), 72-77.

Kable, A. K., Guest, M., \& McLeod, M. (2011). Organizational risk management and nurses' perceptions of workplace risk associated with sharps including needlestick injuries in nurses in New South Wales, Australia. Nursing \& health sciences, 13(3), 246-254.

Kebede, G., Molla, M., \& Sharma, H. R. (2012). Needle stick and sharps injuries among health care workers in Gondar city, Ethiopia. Safety Science, 50(4), 1093-1097.

Kommogldomo, E. D. (2016). Needle stick and sharps injuries among health care workers at the 37 Military Hospital (Doctoral dissertation, University of Ghana).

Kumar, A., Khuwaja, A. K., \& Khuwaja, A. M. (2012). Knowledge practice gaps about needle stick injuries among healthcare workers at tertiary care hospitals of Pakistan. Journal of Ayub Medical College Abbottabad, 24(3-4), 50-52.

Lee, L. K., \& Hassim, I. N. (2005). Implication of the prevalence of needlestick injuries in a general hospital in Malaysia and its risk in clinical practice. Environmental health and preventive medicine, 10(1), 33-41.

Lori, J. R., McCullagh, M. C., Krueger, A., \& Oteng, R. (2016). Sharps injuries among emergency department nurses in one tertiary care hospital in Ghana. International Emergency Nursing, 28, 14-19.

Manzoor, I., Daud, S., Hashmi, N. R., Sardar, H., Babar, M. S., Rahman, A., \& Malik, M. (2010). Needle stick injuries in nurses at a tertiary health care facility. Journal of Ayub Medical College Abbottabad, 22(3), 174-178.
Memish, Z. A., Assiri, A. M., Eldalatony, M. M., Hathout, H. M., Alzoman, H., \& Undaya, M. (2013). Risk analysis of needle stick and sharp object injuries among health care workers in a tertiary care hospital (Saudi Arabia). Journal of epidemiology and global health, 3(3), 123-129.

Muralidhar, S., Kumar Singh, P., Jain, R. K., Malhotra, M., \& Bala, M. (2010). Needle stick injuries among health care workers in a tertiary care hospital of India. Indian Journal of Medical Research, 131(3), 405.

Nsubuga, F. M., \& Jaakkola, M. S. (2005). Needle stick injuries among nurses in sub-Saharan Africa. Tropical medicine \& international health, 10(8), 773-781.

Perry, J., Ginger Parker, M. B. A., \& Jagger, J. (2003). 2003 Percutaneous Injury Rates. Dentist, 2, 0-1.

Prüss-Üstün, A., Rapiti, E., \& Hutin, Y. (2005). Estimation of the global burden of disease attributable to contaminated sharps injuries among health-care workers. American journal of industrial medicine, 48(6), 482-490.

Rais, N., \& Jamil, H. M. (2013). Prevalence of needle stick injuries among health care providers. Int $\mathrm{J}$ Endorsing Health Sci Res, 1(2), 73-9.

Smith, D. R., Mihashi, M., Adachi, Y., Nakashima, Y., \& Ishitake, T. (2006). Epidemiology of needlestick and sharps injuries among nurses in a Japanese teaching hospital. Journal of hospital infection, 64(1), 44-49.

van der Molen, H. F., Zwinderman, K. A., Sluiter, J. K., \& Frings-Dresen, M. H. (2011). Better effect of the use of a needle safety device in combination with an interactive workshop to prevent needle stick injuries. Safety Science, 49(8-9), 1180-1186.

Wilburn, S. Q., \& Eijkemans, G. (2004). Preventing needlestick injuries among healthcare workers: a WHO-ICN collaboration. International journal of occupational and environmental health, 10(4), 451-456. 